\title{
Leukaemia risk and social contact in children aged $0-4$ years in southern England
}

\author{
Eve Roman, Ann Watson, Diana Bull, Krys Baker
}

It has often been suggested that the observed excess of childhood leukaemia around some nuclear establishments might have resulted from unusual patterns of migration and population mixing, affecting the local incidence of

an infectious agent that caused leukaemia. In particular, Kinlen has proposed, "that children in families with a high level of personal contacts may be at a greater risk" and that contacts between parents at work and during the course

Numbers of cases and matched controls* and relative risks (RR) (95\% confidence intervals (CI)) for leukaemia and non-Hodgkin's lymphoma diagnosed before age five years in west Berkshire and north Hampshire during 1972-89 by selected indicators of familial social contact the child's birth and diagnosis

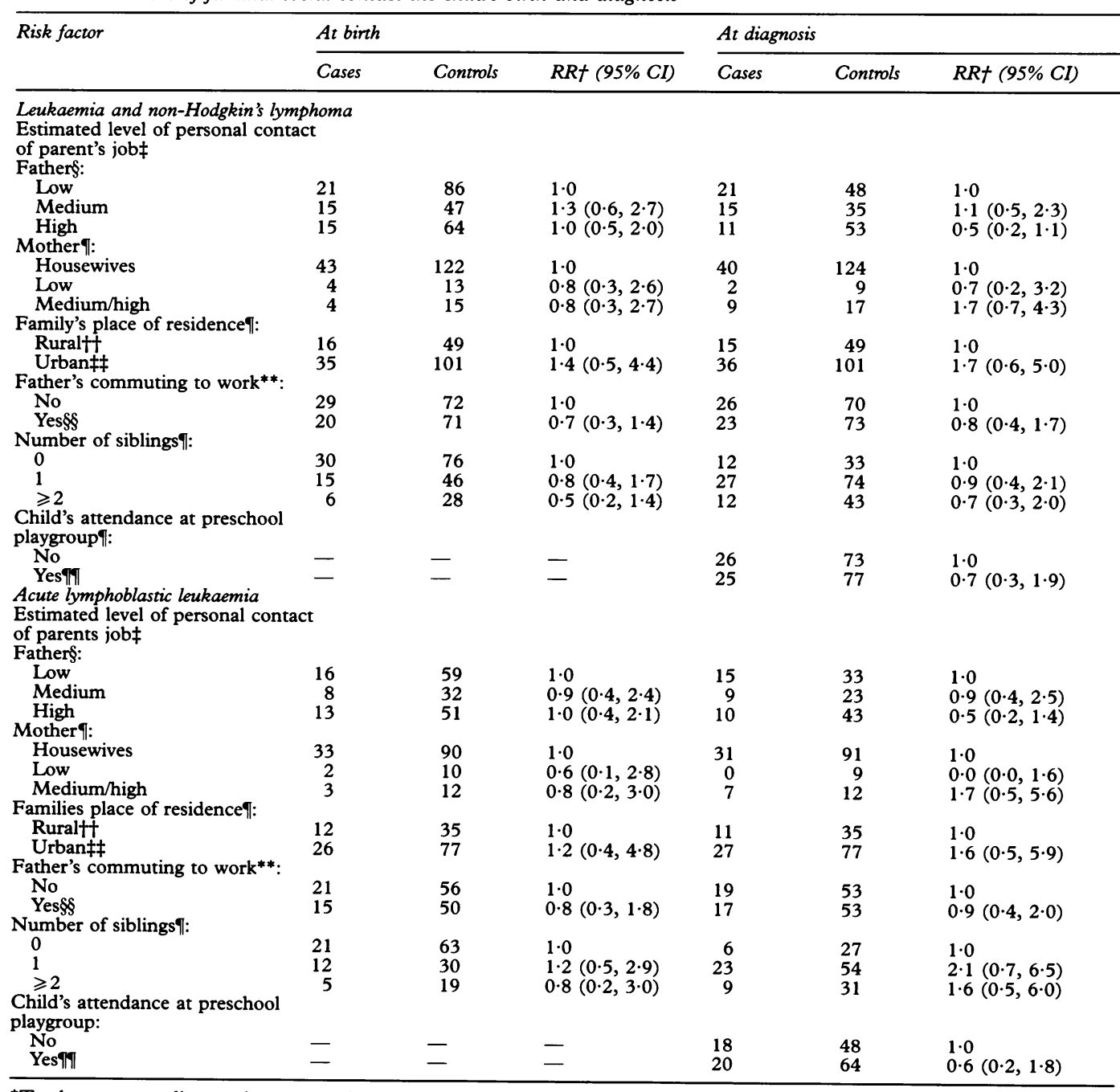

\section{ICRF Cancer Epidemiology Unit, Gibson Building, Radcliffe Infirmary, Oxford OX2 6HE \\ E Roman \\ D Bull \\ K Baker}

Stoke Mandeville Hospital, Department of Haematology, Aylesbury, Bucks HP21 3AL

A Watson

Correspondence to: Dr E Roman, ICRF Cancer Epidemiology Unit, Gibson Building, Radcliffe Infirmary, Oxford OX2 $6 \mathrm{HE}$.

Accepted for publication May 1994

Totals vary according to the source of information used. †Estimated by using informative matched sets and conditional exact methods. ¥Codes from the Office of Population Censuses and Surveys classification of occupations 5 :

High: 1, 9, 17-18, 24, 26, 28, 31-35, 37-43, 45-49, 51-53, 59, 63, 85, 87, 95, 100-106, 108-110, 116-117, 123-125, 127-128, $130-134,137-148,150-157,159-161,163-164,174,210-211,221,249,253,258,260,317-318,320,323,325-326,328,335$ Medium: 2-5, 10, 13-16, 19-23, 25, 27, 29-30, 36, 44, 50, 58, 60-62, 64, 68-69, 82-84. 89-94, 96-97, 111, 113, 126, 129 , $135-136,149,158,162,165,185-187,214,219,247,251,300,302-305,319,324,327,329$

Low: 6-8, 11-12, 54-57, 65-67, 70-81, 86, 88, 98-99, 107, 112, 114-115, 118-122, 166-173, 175-184, 188-209, 212-213, $215-218,220,222-246,248,250,252,254-255,257,259,261-299,301,306-316,321-322,330-334,337-347$

\$Occupation at child's birth as stated on the birth certificate, occupation at child's diagnosis as reported at father's interview

IInformation reported by the mother at interview. **Information reported by the father at interview. HPostcodes RG7-9, 12, 15-20, 25-28, GU34, SO24. ¥¥Postcodes RG1-6, 10-11, 13-14, 21-24 (Reading, Newbury, Basingstoke). \$\$ravelled $20 \mathrm{~km}$ or more to work on a daily basis. 97 Three months or more in the year before diagnosis. 
of their travel to work (commuting) may be important predictors of disease in their children. ${ }^{12}$ To explore this hypothesis, results are presented from a case-control study of leukaemia in children aged 0-4 years, which was conducted in the vicinity of the nuclear establishments at Aldermaston and Burghfield. ${ }^{3}$

Subjects, methods, and results: Details of the subjects and methods have been previously described. ${ }^{3}$ Briefly, the cases comprised 54 children aged $0-4$ years who had leukaemia or non-Hodgkin's lymphoma diagnosed during 1972-89, who were born in west Berkshire or north Hampshire, and were resident there when their cancer was diagnosed. Four controls per case were selected from hospital delivery registers, and each was individually matched to its corresponding case by sex, date of birth (within six months), mother's age (within five years), and area of residence at birth and time of diagnosis. Because the numbers of informative matched sets were small in many of the analyses, $95 \%$ confidence intervals and $\mathrm{p}$ values for relative risks were calculated using conditional exact methods based on the binomial distribution. However, when more than two levels of exposure were analysed, conditional logistic regression was used to ensure that the estimated risks were consistent for different pairs of exposure levels. ${ }^{4}$

The analyses in the accompanying table relate to the $51(94 \%)$ cases whose parents were interviewed, and their corresponding controls. The total number of subjects used in the various analyses differs with the information source employed. In all instances the maximum amount of available data was input for analysis. Findings are shown separately for two points in time, when the child was born and when the child was diagnosed with cancer; and for two disease groupings, all leukaemias and nonHodgkin's lymphomas combined and acute lymphoblastic leukaemia alone. All variables examined and results presented were selected a priori on the basis of previous suggestions. ${ }^{12}$

Comment: No evidence for an association between the development of leukaemia before age 5 years and a family's estimated level of likely social contact were found in the data examined here (table). The parents of children with leukaemia seem neither more, nor less, likely to have had jobs involving high levels of social contact, live in large towns, or commute to work. As well as the analyses shown, other time periods and factors, such as house moves, residence in specific towns, parental job changes, and a detailed examination of jobs likely to involve particularly high levels of personal contact were investigated but no correlations emerged. Information about specific childhood infections, allergies, and immunisations also yielded no notable case-control differences.

Although our findings do not support the proposition that population mixing at the household level is a risk factor for childhood leukaemia, the possibility that a real effect exists cannot be entirely ruled out since the indicators of exposure used are somewhat crude and the confidence intervals are wide. At the community level, a recent ecological study conducted in France has also failed to find evidence of an increased risk of leukaemia in young people living in areas which sustained rapid population growth between 1968 and $1990 .^{6}$

The suggestion that childhood leukaemia has an infectious aetiology is attractive, and there are many features of the disease which accord with this possibility. More analyses of the type presented here and more information about specific infections are clearly required.

We thank Noreen Maconochie for statistical advice.

1 Kinlen LJ, Hudson CM, Stiller CA. Contacts between adult as evidence for an infective origin of childhood leukaemia: an explanation for the excess near nuclear establishments in West Berkshire? Br f Cancer 1991;64:549-54.

2 Kinlen LJ, Stiller C. Population mixing and excess of child hood leukaemia. BMf 1993;306:930

3 Roman E, Watson A, Beral V, et al. Case-control study of eukaemia and non-Hodgkin's lymphoma among children aged 0-4 years living in west Berkshire and north Hampshire health districts. BMF 1993;306:615-21.

4 Breslow NE, Day NE. Statistical methods in cancer research. Vol 1. The analysis of case-control studies. Lyon: International Agency for research on Cancer, 1980: IARC scientific publications no 82

5 Office of Population Censuses and Surveys. Classification of occupations and coding index. London: HMSO, 1980.

6 . com population increase. Br $\mathcal{F}$ Cancer 1994;69:110-13.

\section{Spread of influenza: a study of risk factors in homes for the elderly in Wales}

A Mukerjee

Communicable Disease Control Unit, Mid Glamorgan Health Authority, Abton House, Wedal Road, Cardiff CF4 3QX A Mukerjee

Correspondence to: Dr A Mukerjee. Accepted for publication May 1994
In the winter of 1991-92 there was an outbreak of influenza in the homes for the elderly in Mid Glamorgan. A survey was later carried out to ascertain the risk factors for the spread of infection and their implications on the current policy of influenza immunisation.

Subjects and method: Homes were requested to send the name, age, and influenza vac- cination status of all residents and staff (whole or part time) with the date of onset of influenza defined as "upper respiratory tract symptoms, fever, chills, myalgia, and cough" ${ }^{\text {" between }}$ weeks $91 / 50$ and $92 / 8$. The homes were also asked to provide details of possible risk factors ${ }^{2}$ such as the mean age of the residents, physical characteristics, available facilities, and infection control policy. Paired sera collected 